\title{
How consensus-building conversation changes our minds and aligns our brains
}

3 Beau Sievers ${ }^{1,2}$, Christopher Welker ${ }^{2}$, Uri Hasson ${ }^{3}$, Adam M. Kleinbaum ${ }^{4}$, Thalia Wheatley ${ }^{2,5}$

$4{ }^{1}$ Department of Psychology, Harvard University, Cambridge, MA 02138

${ }^{2}$ Department of Psychological and Brain Sciences, Dartmouth College, Hanover, NH 03755

${ }^{3}$ Princeton Neurscience Institute, Princeton University, Princeton, NJ 08544

${ }^{4}$ Tuck School of Business, Dartmouth College, Hanover, NH 03755

${ }^{5}$ Santa Fe Institute, Santa Fe, NM 87501

Correspondence to: Beau Sievers, 33 Kirkland St, Office 1462, Harvard University, Cambridge, MA 02138. beau@beausievers.com

\section{Abstract}

\section{Introduction}

We take for granted that conversation plays a profound role in shaping belief and coordinating behavior. We rely on consensus-building conversation when the stakes are high, as in jury trials, scientific funding decisions, and elections. Conversation is also pervasive in mundane, day-to-day life, from bars and coffee klatsches to workplace meetings (Mroz, Allen, Verhoeven, \& Shuffler, 2018). Indeed, conversation is the basis of deliberative democracy (Fishkin, 2019), and disruption of public conversation is considered a serious political threat (Lazer et al., 2018). Yet, we lack scientific understanding of how conversation shapes our beliefs and brain responses over time. How does talking change our thinking? 
The internal beliefs that determine our way of seeing the world do not always reflect how we behave towards others-private acceptance is distinct from public compliance (Maass \& Clark, 1983). Psychologists, economists, and others offering explanations of social influence have therefore often treated conversation as a means of achieving public compliance, setting private beliefs aside. This may be because public compliance is easier to measure. In this study, we set out to test whether conversation has the long-lasting power to shape the way a group of people sees the world.

We show that consensus-building conversation can cause groups to share a way of seeing the world by aligning their future brain activity. In the brain imaging studies reported below, conversation aligned brain activity associated with a wide range of cognitive processes, from visual attention to language understanding and memory. Further, we found that some conversations created more alignment than others. Groups with participants perceived as having high social status showed unequal turn-taking and much lower alignment, whereas groups with participants who were more central in their social networks showed more equal turn-taking and greater alignment. This may be because perceived high-status participants signaled disbelief in others' proposals and spoke more, disrupting group consensus. By contrast, high-centrality participants facilitated equal turn-taking and were more likely to adapt their own brain activity to the group. These results have implications for understanding how cognitive processes shape the structure of social networks, as well as for theories of social influence, language and the mind.

\section{Background}

Previous research has shown that conversation determines important attitudes and behaviors. Even brief conversations can have meaningful effects, such as creating support for anti-discrimination laws (Broockman \& Kalla, 2016) or influencing voting behvior (Pons, 2018). Further, these effects can spread through social networks. Talking to a friend about voting may affect not only who they vote for, but who their future conversation partners vote for (Pattie \& Johnston, 2000, 2001). Remarkably, even brief social contact can influence the health outcomes of friends and friends of friends (Christakis, 2004; Smith \& Christakis, 2008), including depression (Rosenquist, Fowler, \& Christakis, 2011), drug and alcohol use (Andrews, Tildesley, Hops, \& Li, 2002; Burt \& Peterson, 1998; Chen, White, \& Pandina, 2001; Mednick, Christakis, \& Fowler, 2010), and obesity (Christakis \& Fowler, 2007). However, it remains unclear whether these conversations shape the way individuals fundamentally see the world, or are merely a means of enforcing public compliance.

Neural alignment can be measured using the inter-subject correlation (ISC) of blood-oxygen level dependent signal from functional magnetic resonance imaging (fMRI) (see Hasson \& Frith, 2016 for a review). Recent studies suggest that neural alignment across people tracks their degree of shared understanding. For example, Chen et al. (2016) used ISC to show that the structure of memory was shared among those with similar experiences. ISC has also been used to draw out inter-individual differences. When some movie-viewing participants pretended to be detectives while others pretended to be interior decorators, the decorators' brains systematically differed from the detectives' brains (Lahnakoski et al., 2014). Likewise, when two groups were given different interpretations of an ambiguous story, alignment of brain activity was higher among people given the same interpretation (Yeshurun et al., 2017). A similar pattern of results held when participants invented their own interpretations of movie 
depicting interacting abstract shapes (Nguyen, Vanderwal, \& Hasson, 2019).

In these prior studies, experimenters either dictated an interpretation to a group or individuals reached an interpretation in isolation, eliminating the natural, back-and-forth dynamic of mutual social influence. Fortunately, studies of brain alignment need not be limited to top-down instruction in artificial settings. For example, brain alignment in the classroom has been associated with student engagement (Dikker et al., 2017) and retention of course content (Bevilacqua et al., 2018). Interestingly, Parkinson and colleagues (2018) found that friends in a real-world social network were more likely to have similar brain activity, suggesting brain alignment may play a role in determining who befriends whom, perhaps in conjunction with social reward systems (Zerubavel, Hoffman, Reich, Ochsner, \& Bearman, 2018). Previous research has also shown that personality traits (Celli \& Polonio, 2013; Liu \& Ipe, 2010; Sasovova, Mehra, Borgatti, \& Schippers, 2010; Staiano et al., 2012) predict network centrality, suggesting that alignment of brain activity within social networks may depend on the social and conversational behaviors of individuals.

Here we use ISC to assess whether consensus-building conversation can align thinking within groups. Further, by using a naturalistic experimental paradigm and drawing participants from real-world social networks, we identify relationships between conversation behavior, network centrality, and neural influence. Although we cannot look at any one person's brain and know the exact contents of their thoughts, we can look at whether activity in conversation partners' brains is similar to one another. This allows us to use neural alignment as a proxy for a shared way of seeing the world. This study is the first to evaluate how face-to-face, open-ended social interactions shape participants' future neural responses to complex, naturalistic stimuli. In particular, this study asks: (1) Can natural, consensusbuilding conversation cause people to share a way of seeing the world? (2) When people share a "way of seeing the world," what are they sharing? In other words: What cognitive processes can be aligned by conversation? (3) Are neurally influential people more central in their social networks? (4) What conversational factors contribute to consensus and neural alignment?

\section{Experimental paradigm}

To answer these questions, we designed an experiment that allowed us to identify changes in brain alignment that could only be caused by conversation. In Session 1, participants ( $N=49$ ) watched movie clips with ambiguous narratives during brain scanning using fMRI. Afterward, participants answered a survey assessing their beliefs about each clip's narrative. In Session 2, participants met in small groups (9 groups; mean group size $=4.2$ ) to discuss the movie clips with the goal of reaching a consensus. Group membership was randomly assigned, pursuant to participants' scheduling constraints. Each group answered the survey presented in Session 1, except expressing the shared view of the group. Participants then rated the influence of the other participants and indicated their personal level of agreement with the consensus. In Session 3, participants re-watched the movie clips during fMRI scanning, along with additional novel clips featuring the same characters. Participants then answered a survey assessing their beliefs about the novel clips. A control group $(\mathrm{N}=9)$ skipped Session 2, doing both fMRI sessions without the intervening group conversation.

To facilitate testing our social network centrality hypothesis, all participants were Master of Business Administration students at a private university in the United States. Because the university was rural 
and relatively isolated, the students formed a tight-knit community and, as part of their coursework, answered a survey used to map their cohort's social network.

The experimental methods were approved by the Dartmouth College Committee for the Protection of Human Subjects and all participants gave written informed consent. For a complete description of the experimental procedure, see Methods.

\section{Results}

\section{Conversation aligned future brain activity}

All participants but two reported agreeing with their group's consensus (as rated from -3 to +3 with values $>0$ indicating more agreement; $\mathrm{M}=1.71 \mathrm{t}(28)=8.32,95 \% \mathrm{Cl}=[1.29,2.13], \mathrm{p}<.001)$. Further, each group converged on a different consensus: after conversation, participants' survey answers became more similar to the answers of their conversation group members, compared to those in other groups (within-group participant pairs: $N=61$, mean distance $=4.61,95 \% \mathrm{Cl}=[3.9,5.31]$; between-group participant pairs: $N=1210$, mean distance $=8.28,95 \% \mathrm{Cl}=[8.11,8.44] ; \mathrm{t}(1269)=-10.08, \mathrm{p}<.001)$ (Supplementary Figure 1). Similarity was measured using city block distance.

A bilateral set of brain regions exhibited conversation-related change in ISC, supporting the hypothesis that consensus-driven conversation can align future brain activity (see Supplementary Material: Cluster reports and Supplementary Table 5 for all fMRI analysis results). Alignment changes took place in different brain areas for different groups discussing different movie clips. Because alignment depends on features of both the group and the conversation, and because conversing groups were small, some brain areas survived multiple comparisons correction across a greater proportion of moviegroup combinations (Figure 1; Supplementary Figure 2). Alignment tended to increase in visual and auditory sensory areas, as well as in higher-order areas associated with the attention and default mode networks, including the temporal parietal junction, angular gyrus, posterior cingulate, medial prefrontal cortex, and temporal pole.

Conversation-related change in ISC was calculated by subtracting ISC before conversation from ISC after conversation, for each pair of participants. Multiple regression with multiple comparisons correction was used to localize change in ISC unique to each movie-group combination, over and above the effect of simply watching the movie clips twice. ${ }^{1}$ A permutation testing method that accounted for the grouped structure of the data was used to limit the false positive rate (G. Chen et al., 2016). Complete regression results are listed in Supplementary Table 5. The scope of inferential statistical generalization is limited to the specific movie clips and groups reported here. See Methods: Conversation-induced change in ISC and Supplementary material: Limitations on generalization.

\footnotetext{
${ }^{1} \mathrm{fMRI}$ regression analyses were controlled by an intercept term corresponding to all within- and between-group participant pairs, including control group participants. This intercept term captured the effect of watching the movie clips twice, with or without conversation, regardless of conversation group. Unless otherwise noted, the reported results are relative to this intercept. Some localized positive changes in ISC were observed for the control group. This may be because some information was shared within most conversation groups, but not among controls, or because simply having a conversation, regardless of content, changed how participants engaged with the movie clips. See Methods: Conversation-induced change in ISC.
} 

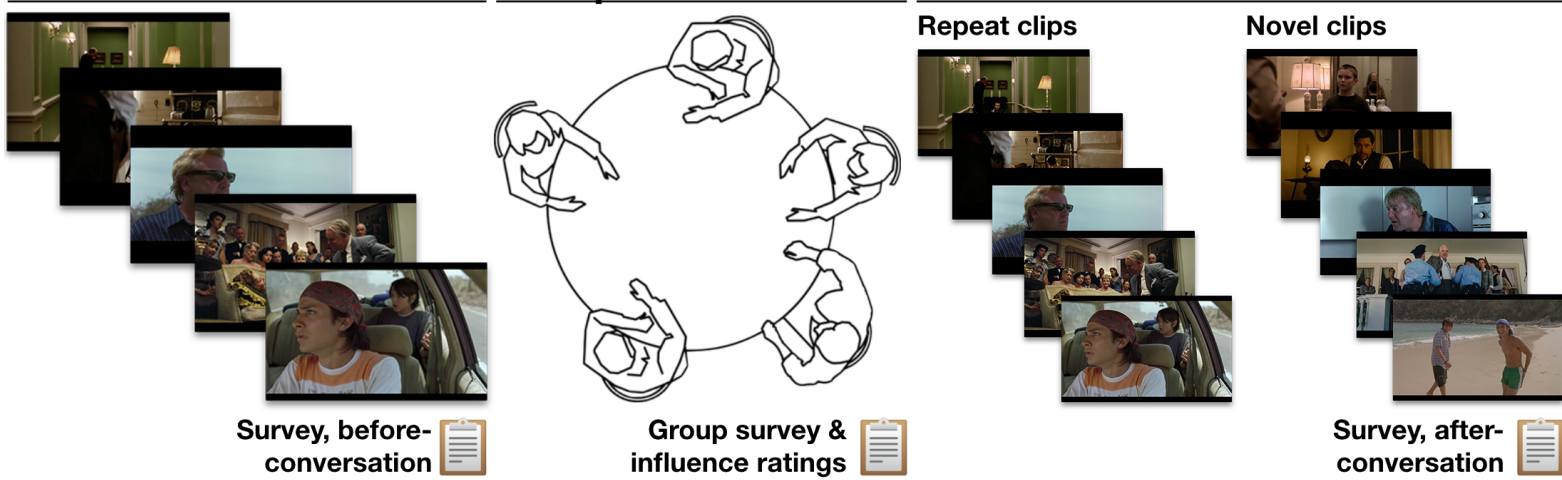
influence ratings

Survey, afterconversation

Change in ISC caused by conversation
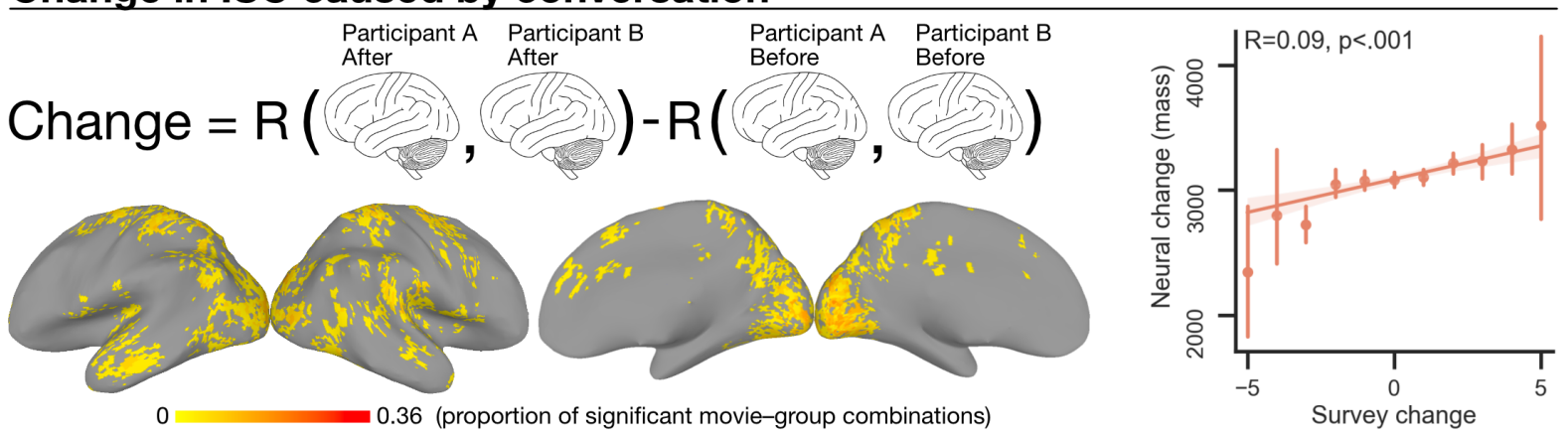

\section{Group-specific ISC during novel clips}

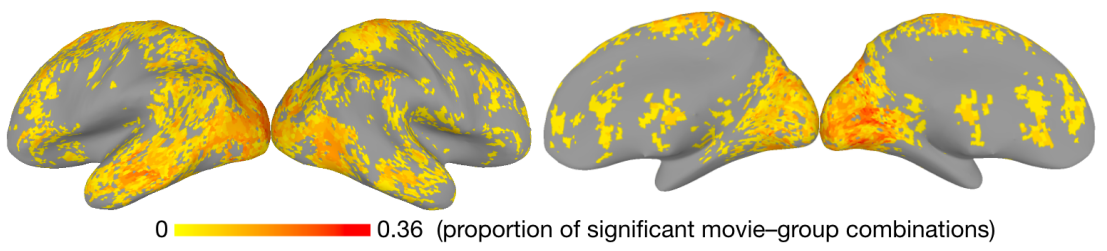

Figure 1: Method and change in alignment results. Top: Participants viewed five ambiguous movie clips during brain scanning, then met in small groups and discussed the movies with the goal of reaching a consensus interpretation. Participants re-watched the movie clips during brain scanning, as well as novel clips from later in each movie. At each step, participants filled out a survey capturing their interpretations. Middle, left: Change in neural alignment caused by conversation (proportion of significant movie-group combinations; reflects both increases and decreases in alignment). Middle, right: Participants whose survey answers became similar showed greater neural alignment. Dots represent the mean mass of the neural change map across participant pairs with the indicated survey change value, where complete behavioral data were available. Vertical lines show 95\% confidence intervals. Diagonal line and shaded region show the regression line of best fit and its 95\% confidence interval. Bottom: Group-specific neural alignment during viewing of novel stimuli that were only shown in fMRI session 2 and were not discussed during conversation.

Participant pairs whose survey answers became similar showed greater whole-brain alignment, across movie clips and groups $(\mathrm{R}=0.09, \mathrm{p}<.001, \mathrm{~N}$ pairs=3478), confirming that neural alignment was driven in part by change in belief (see Supplementary Material: Comparing behavioral and neural results). Whole-brain alignment was calculated as the summed positive mass of unthresholded change in ISC. Note that while most statistically significant regions showed increases in alignment, some regions showed decreases. See Supplementary Material: Cluster reports for brain maps, Neurosynth terms (described below), change in alignment over time, and visualization of group convergence for each significant brain area. 
Importantly, group-specific neural alignment also increased when participants viewed novel clips sampled from the same ambiguous movie (Figure 1, bottom). Multiple regression was used to localize brain areas where ISC was uniquely predicted by conversation for each movie-group combination (see Methods: Conversation-induced change in ISC).

\section{Conversation aligned a range of cognitive processes}

Conversation aligned brain areas associated with a wide range of cognitive processes, as estimated by quantitative reverse inference using Neurosynth (Yarkoni, Poldrack, Nichols, Van Essen, \& Wager, 2011). Neurosynth uses a large database of previously published brain imaging literature, where Englishlanguage terms are associated with brain activation likelihood maps. For each statistically significant brain area in all reported fMRI analyses, we estimated which Neurosynth terms were a likely match. We then identified groups of terms that tended to co-occur using principal component analysis. 18 groups of terms explained $70 \%$ of the variance in the term probability data (Figure 2 ). The group of terms that explained the most variance included words related to vision and motion, suggesting that one important function of conversation is future alignment of visual attention. Other high-ranking principal components were associated with motor activity, working memory, face perception, the default network, auditory perception, body movement and social understanding, and language. See Supplementary Material: Cluster reports for term probabilities for each brain region and Methods: Reverse inference with Neurosynth for additional detail.

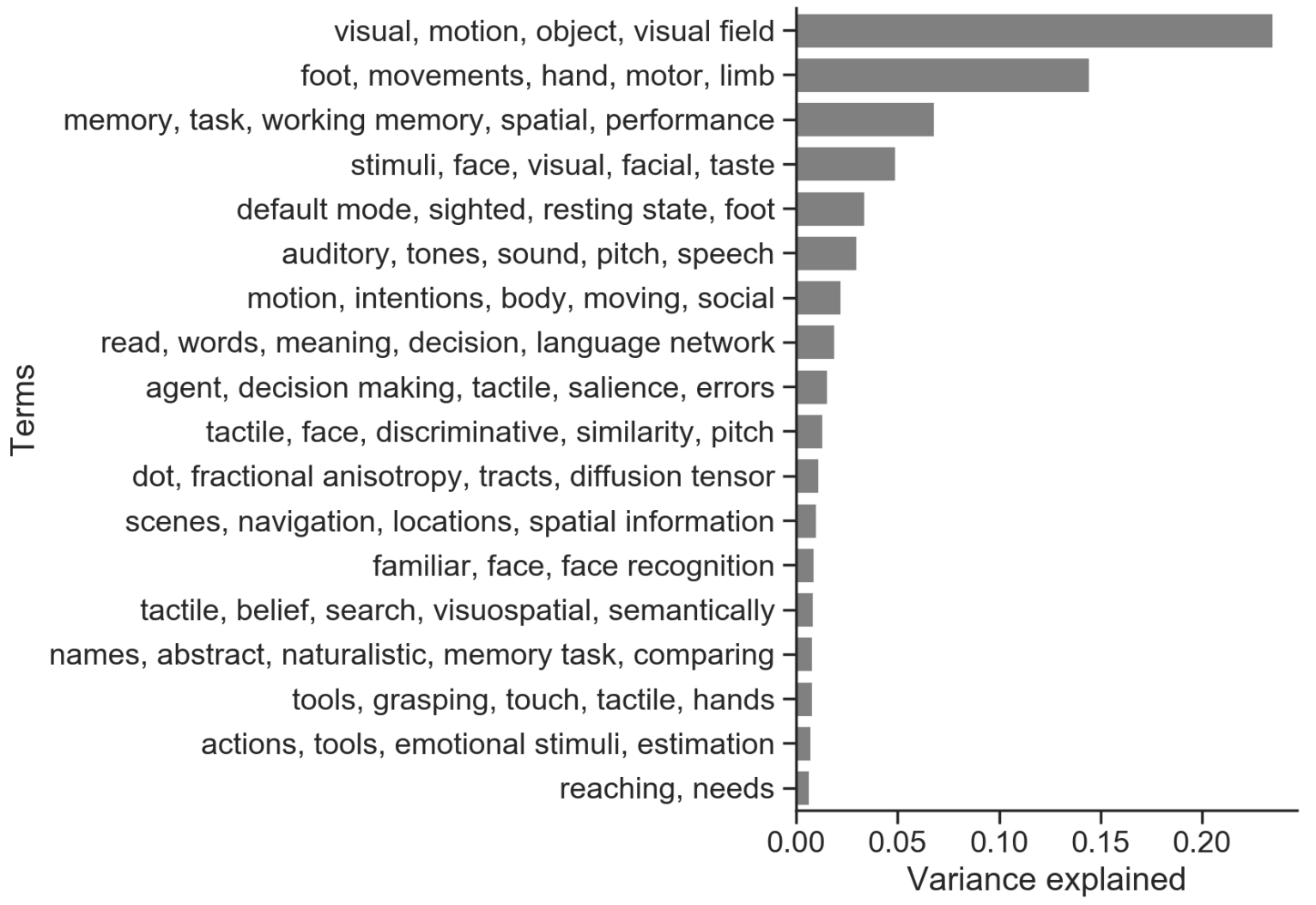

Figure 2: Conversation aligned brain areas associated with a range of cognitive processes, as identified by quantitative reverse inference using Neurosynth (Yarkoni et al., 2011). 


\section{Neural influence and social network centrality}

The change analysis reported above could not capture directional social influence. Consider the following hypothetical example: Amar influences Beth, and later in the conversation Carlos influences Amar. The method used above would detect that Carlos and Amar had aligned, but would overlook Amar's influence on Beth. To check the degree to which each participant influenced each other participant we performed a neural influence analysis. Each participant was analyzed as both the person exerting influence (called the ego) and the person being influenced (called the alter), accounting for the possibility that participants could both influence others and be influenced by others in different respects. The amount of neural influence was the extent to which the alter became more similar to the ego's initial pattern of brain activity. Neural influence maps were calculated by comparing the brain activity of the ego before conversation to that of the alter both before and after conversation. Whole-brain neural influence was calculated as the summed positive mass of the unthresholded neural influence maps.

Participants' centrality in the social network of their school cohort was assessed using brokerage and eigenvector centrality. Brokers are those who connect people who would not otherwise be connected, allowing them to control the flow of information between cliques (Burt, 1992). People with high eigenvector centrality are both well-connected and have many well-connected friends (Bonacich, 1972) (Figure 6). Because brokerage and eigenvector centrality were correlated in our population, we also computed a PCA centrality score capturing variation common to both measures (see Methods: Social network centrality). P-values reported below were calculated using permutation testing to account for network autocorrelation.

Surprisingly, participants who were central in their social networks were more likely to be influenced by others: Alter PCA centrality was correlated with whole-brain influence $(R=0.1, p=0.021, N=560)$. But highly central participants were not more likely to exert influence on others: Ego PCA centrality was not significantly correlated with whole-brain influence $(R=-0.06, p=0.145, N=560)$. Because of hypothesized differences between brokerage and eigenvector centrality (e.g., Burt, 1992, 2010), we assessed the unique contributions of each centrality measure using multiple regression. Predictors included eigenvector centrality and brokerage for both ego and alter, controlling each variable against the others. This regression model modestly predicted whole-brain influence $(F(555,4)=5.02, p=0.046)$, despite the collinearity of eigenvector centrality and brokerage. Alter eigenvector centrality was associated with more whole-brain influence $(b=335.3, p<.001)$, while alter brokerage was associated with less whole-brain influence $(b=-270.3, p=0.004)$. Given that neural influence depends on alter PCA centrality, this result suggests brokers are somewhat less likely to be influenced by others than those with high eigenvector centrality. No ego centrality measures significantly predicted whole-brain influence.

Neural influence occurred in different parts of the brain for different pairs of participants. To account for these differences, multiple regression was used to localize network centrality-dependent neural influence in specific brain areas. See Methods: Neural influence for a detailed description of the regression model and Supplementary Table 5 for all fMRI analysis results. Centrality-dependent influence affected a broad set of brain regions for both egos and alters, showing that high-centrality participants were both more neurally influential and more likely to be neurally influenced by others in 

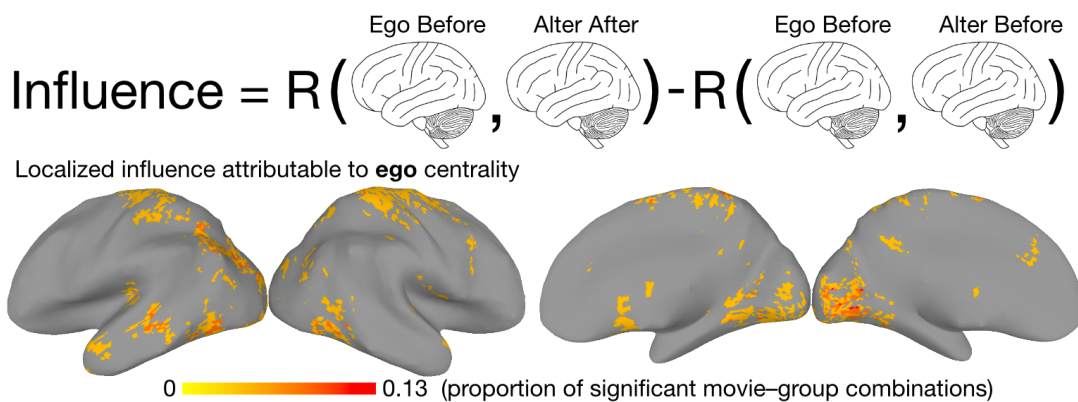

Localized influence attributable to alter centrality

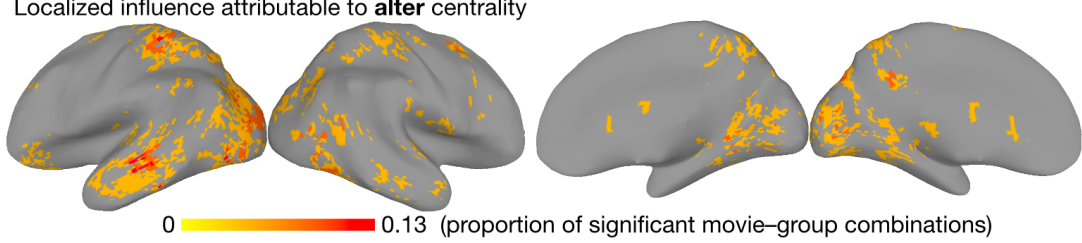

Whole-brain influence

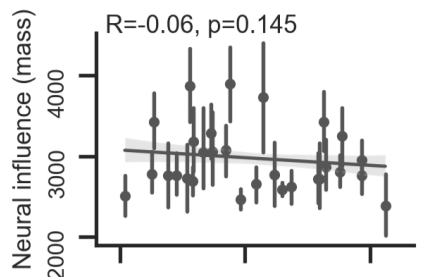

Ego PCA centrality

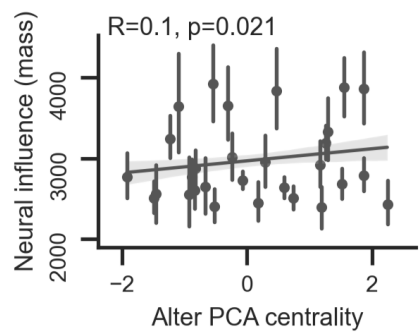

Figure 3: Neural influence results. Left: Neural influence attributable to participants' network centrality. For each participant pair, one was designated the ego while the other was designated the alter. Neural influence reflects the movement of the alter's BOLD time series toward the ego's initial BOLD time series. Right: At the whole-brain level, highly-central participants were more neurally influenced by others. Dots represent the mean mass of the neural influence map across participant pairs where the ego (top) or alter (bottom) had the indicated PCA centrality value, where the pair conversed in the same group and where complete behavioral data were available. Vertical lines show 95\% confidence intervals. Diagonal lines and shaded regions show regression lines of best fit and their $95 \%$ confidence intervals.

Confirming that neural influence was related to changes in belief, survey influence calculated over the survey answers of ordered participant pairs was correlated with whole-brain neural influence $(R=0.06$, $\mathrm{p}<.001, \mathrm{~N}$ pairs $=8620$ ) (see Supplementary Material: Comparing behavioral and neural results).

\section{Network centrality and perceived social status affected group alignment}

Some groups showed stronger consensus and greater neural alignment than others. Exploratory analysis showed that groups that spoke more had more neural alignment $(b=3.52, p=0.007)$ and this effect was stronger for groups where conversation turn-taking was more equal among the participants (2-way interaction, $b=-1.34, p=0.038$ ). However, groups with perceived high-status participants (as judged by hypothesis-blind raters) showed less neural alignment as more words were spoken in conversation (2-way interaction, $b=-7.39, p=0.021$ ). By contrast, groups with high-PCA centrality participants showed more neural alignment $(b=1.33, p=0.025)$.

Below, we describe similarities and differences between high-centrality and high-status participants in their word choices and general conversation behaviors. Here, "centrality" refers to PCA centrality and "status" refers to perceived social status as judged by hypothesis-blind raters. All analyses reported in this section were exploratory. For further description and examples of participants' conversation behavior, see Supplementary Material: What did participants discuss in conversation? See https: //osf.io/krgfb/ for conversation transcripts.

Word use in each speech turn was modestly predictive of participants' PCA centrality $\left(R_{a d j}^{2}=0.04, \mathrm{~F}(350\right.$, 
$7771)=2.0, \mathrm{p}<.001)$ and perceived status $\left(R_{a d j}^{2}=0.06, \mathrm{~F}(350,7771)=2.39, \mathrm{p}<.001\right)$. For regression analysis details see Methods: Conversation content analysis; see Supplementary Table 3 for word-by-word betas and p-values.

To qualitatively understand how the identified words functioned in context, we searched the conversation transcripts and read the speech turns before, after, and containing each word. This revealed marked differences in how high-centrality and high-status participants' behaved in conversation. Highcentrality participants encouraged others to express themselves, whereas high-status participants did the opposite.

While both high-centrality and high-status participants used casual language like cool, gotta, for sure, dude, shit, gonna, and_'cause,_these words were stronger predictors of centrality than status. High-centrality (but not high-status) participants also spoke quite frankly, discussing sexual content ("There's gonna be so much tension it's gonna turn sexual"), describing ideas or movie clips as weird ("I had some really weird thoughts on this one"), and using fuck as an intensifier ("Weird fucking movie"). High-centrality participants also prompted others to comment on the group's survey answers using far ("So, what I have so far is..."), answer ("That's a good answer now"), and character (Reading a survey question aloud: "Are the characters discussing politics?"). These behaviors may have made other participants more comfortable expressing themselves. By contrast, high-status (but not highcentrality) participants challenged others' proposals by asking really? (e.g.: Speaker 1: "Yeah, that's Joaquin Phoenix." Speaker 2: "Really?"). They also tended to express tentative or reserved approval of others' proposals using fine ("I can go with it. I'm fine."). These behaviors may have made others less comfortable expressing themselves.

Pairs of hypothesis-blind coders classified each speech turn by its function in the conversation (see Methods: Conversation content analysis). Both high-status and high-centrality participants were more likely to direct attention to other speakers (status: $b=0.21, p<.001$; centrality: $b=0.19, p<.001$ ) and to propose explanations of movie clip content (status: $b=0.05, p<.001$; centrality: $b=0.03, p=0.009$ ). Speech turns by both high-status and high-centrality participants were ignored slightly less (status: $b=-0.03$, $p<.001$; centrality: $b=-0.01, p=0.013)$. Interestingly, both high-status and high-centrality participants were more likely to reject others' proposed explanations. However, high-status participants favored implicit rejection (e.g., by asking "Really?" or saying something that when evaluated in context suggests another's proposal is unlikely) ( $b=0.13, p=0.022)$ while high-centrality participants favored explicit rejection (e.g., by saying "No" or "That's not true" and possibly providing an alternate explanation) $(b=0.1, p=0.024)$.

High-status participants (but not high-centrality participants) were more likely to request clarification from other speakers $(b=0.14, p=0.005)$, to give orders to other group members $(b=0.14, p<.001)$, and to direct attention to specific movie content $(b=0.13, p<.001)$. They were more likely to speak with confidence $(b=0.03, p<.001)$, and more likely to have their proposals incorporated into the group explanation of the movie content (but note that this does not require private acceptance by others) $(b=0.03, p<.001)$.

High-status participants spoke more $(\mathrm{R}$ (status, words spoken $)=0.65, \mathrm{p}<.001)$ and were rated as more influential by others in their group $(R$ (status, influence rating $)=0.74, p<.001)$. Despite this, high-status was negatively correlated with pairwise, whole-brain neural influence $(R($ status, neural influence)=- 
$0.05, p<.001)$. Unlike high-status participants, high-centrality participants did not speak more than others. So while high-status participants may have limited others' contributions, high-centrality participants did not. High-centrality participants were not rated more influential by their group members ( $R$ (PCA centrality, influence rating $)=0.15, p=0.429)$, although participants who spoke more were $(R$ (words spoken, influence rating $)=0.77, p<.001)$.

Behavioral alignment was also affected by high-status and high-centrality participants. The presence of high-centrality participants partially mitigated the effect of unequal turn-taking. Groups with highcentrality participants and a higher Gini coefficient of words spoken showed more agreement in survey responses (2-way interaction, $\mathrm{b}=2.39, \mathrm{p}=0.037$ ). By contrast, groups with both high-centrality participants and high-status participants and a higher Gini coefficient of words spoken showed markedly less agreement in survey responses (3-way interaction, $b=-24.39, p=0.022$ ).

Taken together, these results suggest that the conversation behaviors of high-centrality participants supported group alignment, while the behaviors of participants with perceived high social status produced public compliance without private acceptance, and that this was detrimental to group alignment.

\section{Discussion}

The true-to-life, open-ended study design, in which a group of people tried to make sense of a movie they just watched, exposed the power of conversation to shape how our brains process the world. Cognition does not materialize in isolation, as the thoughts and actions of others continuously shape how we think and act. The present study documents, for the first time, that consensus-building conversation strengthens the neural alignment of group members across a wide range of brain areas. These areas were associated with many cognitive processes, ranging from vision and audition to attention, language, and memory. Each group aligned in its own way, and group alignment was correlated with the degree of behavioral consensus. Further, increases in group alignment generalized to novel stimuli not discussed, indicating that conversation provided a mental framework to interpret new information.

We did not expect all conversations to be equally successful, and some conversation groups aligned more than others. Specifically, groups with participants perceived as having high social status showed less alignment, while groups with high-centrality participants showed more alignment. This may be because high-status and high-centrality participants behaved differently in conversation.

Participants perceived as high in social status spoke more, gave more orders, and implicitly rejected others' proposals. Despite being rated more influential by their groups, these high-status participants were less neurally influential, raising the possibility that their conversation behaviors produced public compliance without private acceptance. This misperception of status cues as markers of influence may play a pernicious role in the reinforcement of power hierarchies (see e.g., Magee \& Galinsky, 2008). By contrast, groups with high-centrality participants had more equal turn-taking, and highcentrality participants did not speak more than others. These participants were both more neurally influential and more likely to be neurally influenced by others-accordingly, their groups achieved greater neural alignment. High-centrality participants may have facilitated this alignment by creating 

a psychologically safe environment, encouraging others to speak (Edmondson \& Lei, 2014), privately accepting and internalizing others' proposals, and rallying their groups around agreeable consensus positions.

High-centrality participants flexibly adapted their brain activity to their groups. It may be that this flexibility directly supports conversational skills that facilitate social connection. Previous research on personality and social network centrality points in this direction: People with high self-monitoring personalities (i.e., those who adapt their behavior to the people around them) tend to be more socially central (Fang et al., 2015; Mehra, Kilduff, \& Brass, 2001), and they become so by making friends across disconnected cliques (Sasovova et al., 2010). Further, survey measures of self-monitoring, cognitive flexibility, and communication flexibility are closely related and highly correlated (Chesebro \& Martin, 2003; Martin \& Rubin, 1995). Although we do not know how our participants became central in their social networks, it is plausible that cognitively flexible, consensus-building approaches to conversation enabled them to grow large and diverse groups of friends. ${ }^{2}$ By connecting the dots between how people think, how people speak, and who they socialize with, we hope to provide a cognitive, process-level view of social network structure.

As across the sciences, arts, and humanities, knowledge is increasingly produced by collaborative, conversing teams (Wuchty, Jones, \& Uzzi, 2007), these effects have practical implications for leadership and management, as well as for predicting the spread of information through social networks. The present results also fit research showing that powerful individuals can afford to manage the conflicts of others, resulting in a benefit to their group (Brush, Krakauer, \& Flack, 2018; Flack, Waal, \& Krakauer, 2005).

Importantly, conversation participants were given the explicit goal of coming to a consensus. While many natural conversations share this feature explicitly (e.g., jury deliberations) or implicitly (e.g., meetings at work), many other important conversation contexts are naturally adversarial (e.g., political debates), and in these contexts we should expect different results. In particular, responsiveness to neural influence may be less desirable in contexts where groups are seeking out a correct solution to a well-defined problem, or where there is direct competition within or between groups. Further, our participants were all Master of Business Administration students at a private university in the United States, and likely differ from the general population in socioeconomic status, general intelligence, and conversation behavior, limiting the generalizability of the reported findings. Uncovering generalizable factors affecting neural alignment will require future research. For further discussion see Supplementary Material: Limitations on generalization.

\section{Theoretical implications: influence, language, and the mind}

In agreement with prior studies (e.g., Hasson, Malach, \& Heeger, 2010; Huth, Heer, Griffiths, Theunissen, \& Jack, 2016), we found that the cognitive processes underlying social understanding were shared across people. Further, we found that these processes were aligned by natural conversation, not only when participants were listening to each other, but into the future. These results bear on theories of social influence, language and the mind.

${ }^{2}$ N.B.: Although Burt $(1992,2010)$ hypothesized that similar advantages would accrue to network brokers, the present results were not specific to brokerage. 
Much research on social influence has focused on public compliance, setting aside the long-lasting effects of social interaction on private cognition (Festinger, 1950; Hornsey, 2008; Kirchler \& Davis, 1986; Maass \& Clark, 1983; Petty \& Cacioppo, 1986; Tajfel, Billig, Bundy, \& Flament, 1971). ${ }^{3}$ However, the present results show that consensus-building conversation aligns neural responses within groups, and that this alignment generalizes to novel stimuli that were not discussed. This suggests a stronger role for private acceptance in understanding social influence, and demonstrates the feasibility of fMRI as a tool for measuring changes in private thought.

Further, this study demonstrates the power of words to shape how our brains process the world. The observed results could only have occurred if participants' ways of speaking were commensurable, complicating strong versions of accounts on which people regularly use the same words but with completely different meanings (Feyerabend, 1987; Lakoff \& Johnson, 1980; Putnam, 1988). Additionally, we would not have observed neural alignment if people tended to represent the same concepts using different neural processes (Fodor, 1974; Putnam, 1967).

Finally, conversation-related neural alignment poses a challenge to the claim that language did not evolve for communication, but instead for organizing individual thought (Berwick \& Chomsky, 2015). By contrast, the reported results are consistent with the theory that one important evolutionary function of language is the coordination of belief (Brennan \& Clark, 1996; Clark, 1996; Clark \& Brennan, 1991; Garrod \& Pickering, 2009; Tylén, Weed, Wallentin, Roepstorff, \& Frith, 2010; Wilkes-Gibbs \& Clark, 1992; Wilson \& Sperber, 2002), and further suggest alignment of neural processes governing attention as a mechanism. The tight, ever-evolving coupling between your thoughts and my thoughts, corresponding to tight neural alignment across our brains, constitutes a mechanism for building group realities based on shared language. This view is compatible with predictive processing accounts of cognition (Clark, 2013; Friston, 2010), especially those that place special importance on the challenge of predicting other people (Lupyan \& Clark, 2015; Theriault, Young, \& Barrett, 2020). The aligning function of conversation may support cumulative development on an evolutionary time scale, as the language used by one generation scaffolds the learning of the next (Dean, Kendal, Schapiro, Thierry, \& Laland, 2012; Hasson, Nastase, \& Goldstein, 2019; Laland et al., 2015; Sterelny, 2007, 2010).

On this account, being a competent language user does not consist primarily in mastery of syntactical rules for building hierarchically structured abstract expressions. Rather, competence consists in internalizing normatively consistent relations between speech acts and neural processes. A word has meaning by virtue of the reliability of its hooks into a shared neural and cognitive infrastructure. Understood in these terms, conversation is a neuro-feedback process (Taschereau-Dumouchel, Cortese, Lau, \& Kawato, 2020), where we read others' neural and cognitive states from traces in their speech, and produce utterances that encourage or constrain those processes, all while allowing others to do the same to us.

\footnotetext{
${ }^{3}$ Deutsch \& Gerard (1955) draw a related distinction between normative and informational social influence: Normative influence occurs when people conform to a group, whereas informational influence occurs when beliefs are revised in light of new information. Although public compliance implies normative influence, normative and informational factors are confounded in the reported paradigm, preventing separate examination.
} 


\section{Methods}

\section{Participants}

Participants who completed the social network survey were first-year Master of Business Administration students at a private university in the United States $(\mathrm{N}=865)$. The survey was completed as a part of their coursework on leadership. A subset of these participants $(N=49)$ went on to participate in the fMRI studies. Scheduling was facilitated by a custom web application that allowed participants to select available sessions. Participants met in small groups with 3-6 participants per group (9 groups; mean group size=4.2). 9 participants were assigned to a separate control group that did not complete the conversation task. All participants provided informed consent, and all experimental procedures were reviewed and approved by the Dartmouth College Committee for the Protection of Human Subjects.

\section{Exclusions and missing data}

A total of 59 participants underwent fMRI scanning, however, 10 participants were excluded, yielding 49 participants. 5 participants were excluded because of technical difficulties during scanning, 1 was excluded because the scanner compatible glasses were insufficient and they couldn't see actors' facial expressions, 2 were excluded because they terminated the scan session due to discomfort, 1 was excluded because they were absent from the group discussion, and 1 was excluded because an anatomical anomaly was detected (this participant was referred to a neurologist for follow-up in accordance with Dartmouth Brain Imaging Center safety policies). Due to a technical error, three groups were given an incorrect version of the survey for fMRI session 2 (after conversation) that did not include run-by-run agreement ratings or yes-or-no questions for the repeated movie clips. These same three groups were also not given pen-and-paper surveys after the group session, but instead verbally confirmed that they agreed with the group consensus (the realization that this was inadequate led to the introduction of the pen-and-paper survey).

\section{Session 1: Before-conversation movie viewing}

Participants viewed a selection of movie clips during fMRI scanning. Naturalistic, narrative movie clips were selected to capture the audience's attention over an extended period of time, maximizing the anatomical extent of neural alignment (Honey et al., 2012). The clips contained interesting but ambiguous social content, and were selected from a range of major motion pictures (listed below in Movies). All movie clips were ambiguous in nature, allowing participants to form a range of possible interpretations that could be updated via social influence. In addition, the sound was turned off, removing music, dialog, and context cues that might otherwise constrain interpretation of the narrative. Two additional movie clips with interesting social content were selected for use in hyperalignment (Bialic, Blichfeld, \& Sinclair, 2016; Pollack, Samuels, Fox, Orent, \& Gilroy, 2007). Hyperalignment clips were presented with the sound on. See Supplementary Table 1 for titles and presentation order. All movie clips were edited for time and narrative continuity. The edited clips may be downloaded at https://osf.io/krgfb/ 
The before-conversation fMRI session consisted of an anatomical scan, five echo-planar imaging (EPI) runs of ambiguous movie clip viewing, and two EPI runs of hyperalignment movie clip viewing (naturalistic movies with rich social interaction, with the sound turned on). After scanning, participants answered a detailed survey to assess their individual, before-conversation understanding of the movie clip content. Questions about each movie clip were accompanied by an array of screenshots to act as a memory aid.

\section{Session 2: Group conversation}

Participants met in groups of three to six, and sat around a circular table. The only instructions were to talk with the goal of reaching a group consensus, and to fill out the survey so it reflected that consensus. The survey, including an array of screenshots for each clip as a memory aid, was presented on a laptop that could be moved around from participant to participant. Participants were allotted 15 minutes of conversation per movie clip, and given verbal warnings when the remaining time reached five minutes and one minute. If participants finished before the time limit was reached, they informed the experimenter and moved directly on to discussing the next clip. After participants were finished, they each went into separate rooms and filled out pen-and-paper surveys where they rated their agreement with the group consensus and evaluated the influence of each group member (including themselves) on the group consensus.

\section{Session 3: After-conversation movie viewing}

In the final fMRI scan session, participants viewed the same selection of clips presented during Session 1. Participants then viewed five more movie clips, each of which was a scene from later in each of the movies they had already seen. These clips were used to test the generalization of the group consensus to novel stimuli. After leaving the scanner, participants answered a survey containing the same questions as in Sessions 1 and 2, except for the new set of clips the participants had just seen.

\section{Movies}

Gower, L., Morris, N., Piel, J-L. (Producers), \& Glazer, J. (Director). (2004). Birth. United States: New Line Cinema.

Pitt, B., Gardner, D., Scott, R., Daly, J., Valdes, D. (Producers), \& Dominik, A. (Director). (2007). The Assassination of Jesse James by the Coward Robert Ford. United States: Warner Bros. Pictures.

Thomas, J. (Producer), \& Glazer, J. (Director). (2000). Sexy Beast. United Kingdom, Spain: Fox Searchlight Pictures.

Sellar, J., Lupi, D., Anderson, P. T., Ellison, M. (Producers), \& Anderson, P. T. (Director). (2012). The Master. United States: The Weinstein Compay.

Cuarón, A., Vergara, J. (Producers), \& Cuarón, A. (Director). (2001). Y tu mamá también. Mexico: 20th Century Fox, IFC Films. 


\section{Hyperalignment movies}

Bialic, G. (Producer), Blichfeld, K., \& Sinclair, B. (Directors). (2016). "High Maintenance” Tick. United States: Home Box Office.

Pollack, S., Samuels, S., Fox, J., Orent, K. (Producers), \& Gilroy, T. (Director). (2007). Michael Clayton. United States: Warner Bros. Pictures.

\section{fMRI image acquisition}

Participants were scanned at the Dartmouth Brain Imaging Center using a 3T Siemens Prisma scanner with a 32-channel head coil. A high resolution T1-weighted MPRAGE anatomical scan (2.32ms TE; $2300 \mathrm{~ms}$ TR; .9x.938x.938mm resolution) was performed the beginning of each scanning session. Functional images were acquired using an echo-planar sequence ( $32 \mathrm{~ms} \mathrm{TE} ; 727 \mathrm{~ms}$ TR; $53^{\circ}$ flip angle; $3 \times 3 \times 3 \mathrm{~mm}$ resolution). The number of scans per run varied depending on the stimulus presented. Sound was delivered using an in-ear headphone system. Foam padding was placed around participants' heads to minimize motion.

\section{fMRI image preprocessing}

See Figure 4 for a schematic describing anatomical and EPI preprocessing.

Anatomical images were deobliqued using AFNI 3dWarp. Brain extraction was performed using ANTs antsBrainExtraction. sh with priors derived from the MICCAI 2012 Multi-Atlas Challenge Data (Landman \& Warfield, 2012). ${ }^{4}$ Tissue segmentation was performed using FSL fast, and tissue masks were saved for calculating tissue-specific nuisance variables (below). Anatomical images were normalized to the non-linear, asymmetrical MNI ICBM152 template (Fonov et al., 2011) using ANTs antsRegistrationSyN. sh, and transformation matrices were saved for normalizing EPI time series (below).

EPI images were motion corrected using FSL mcfli rt and motion outliers (framewise displacement $>$.9) were detected using FSL fsl_motion_outliers. Motion parameters and outlier TR indices were saved. EPI-to-anatomical transformations were calculated using AFNI align_epi_anat.py and saved for normalizing EPI time series (below). EPI images were deobliqued using AFNI 3dWarp, then percentage-scaled (Chen, Taylor, \& Cox, 2016) using NumPy, then normalized to the non-linear asymmetrical MNI ICBM-152 template by concatenating the EPI-to-anatomical and anatomical-toMNI transformations and applying them in a single step using ANTs antsApplyTrans forms. EPI images in MNI space were then iteratively blurred until reaching a smoothness of $6 \mathrm{~mm}$ full width at half maximum using AFNI $3 \mathrm{~dB}$ LurToFWHM. Nuisance variables were removed from the smooth EPI images in MNI space using Nilearn clean_img(). Nuisance regressors included: 6 motion parameters, framewise displacement outliers (one binary regressor per outlier), tissue confounds as described by Simony et al. (2016), linear and quadratic trends, and an intercept term.

\footnotetext{
${ }^{4}$ Available at: https://my.vanderbilt.edu/masi/workshops/
} 


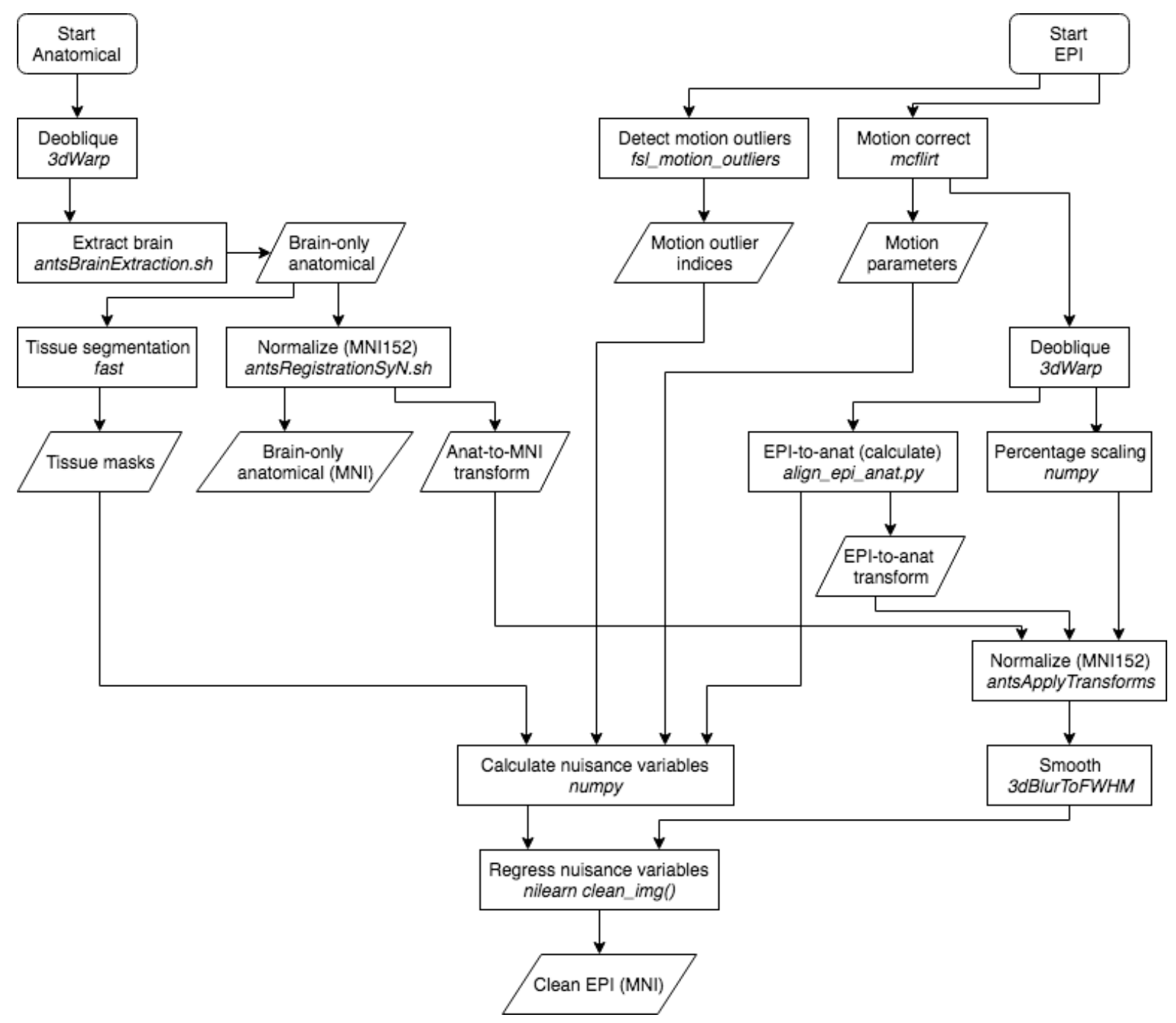

Figure 4: Schematic summary of fMRI preprocessing pipeline.

\section{Hyperalignment}

Searchlight hyperalignment (Guntupalli et al., 2016) was performed using PyMVPA (Hanke et al., 2009). Hyperalignment maps were calculated using runs 6 and 7 from the pre-conversation fMRI session. Because 6 participants did not complete both runs, a second set of hyperalignment maps was calculated using only run 6 , which was completed by all participants. For all analyses, EPI images hyperaligned using both runs 6 and 7 were used if they existed; if not, the EPI image hyperaligned using only run 6 was used. To work around a bug in PyMVPA, ${ }^{5}$ very low-amplitude Gaussian noise was added to invariant features (voxels with no variance) before calculating the hyperalignment maps, and also before forward-mapping of EPI images. Inspection of the images showed that the invariant features were voxels included in the MNI ICBM-152 template mask, but not in individual participants' EPI images, because sometimes parts of the cerebellum were not imaged.

\footnotetext{
${ }^{5}$ Described at: https://github.com/PyMVPA/PyMVPA/issues/589
} 


\section{Inter-subject correlation}

The basic unit of the reported neuroimaging analyses is inter-subject correlation (ISC). Here, ISC is defined as the Pearson correlation of time series in corresponding voxels in a pair of participants. Voxel-wise ISC was calculated on the hyperaligned images using AFNI 3dTcor relate.

\section{Conversation-induced change in ISC}

For each fMRI session, ISC was calculated for every participant pair, creating an ISC matrix. A change matrix was calculated by subtracting before-conversation ISC from after-conversation ISC. The effect of group membership on change in ISC was quantified using regression analysis. Predictor matrices were created for each group, with 1s where both participants were in the target group and 0s otherwise. An all-1s intercept matrix was used to capture change that occurred whether or not participants were in the same group (i.e., change caused simply by watching a movie clip twice). Unraveled vector versions of these matrices were used as predictors in a multiple regression with the unraveled change matrix as the target, executed using NumPy lingalg. lstsq (). The beta values from this regression represent the change in ISC unique to each group. See Figure 5 for a worked example. Repeating this procedure for each movie clip across every voxel in the fMRI data yielded change maps showing where in the brain change occurred for each movie-group combination. See Results: Conversation aligned future brain activity and Figure 1.

To test whether conversation-related change extended to the novel stimuli shown in the afterconversation $\mathrm{fMRI}$ session, a regression was performed using the same predictors, but with the after-conversation ISC matrix as the target.

Significance values were calculated using subject-wise permutation testing (G. Chen et al., 2016). The regression described above was repeated 2000 times. Each time, the rows and columns of the change matrix were identically permuted (Figure 5 , bottom). This procedure satisfies the exchangability assumption of permutation testing, appropriately limiting the false positive rate. Two-tailed $p$-values for each parameter were obtained by calculating the proportion of permutations where the true result was more extreme than the permuted results. Statistical significance was achieved when the true result was more extreme than $97.5 \%$ of the permuted results, establishing a two-tailed alpha value of 0.05 . This approach controls the false positive rate given the pairs-and-groups structure of the study, but it is not equivalent to the use of a multilevel model. See Supplementary Material: Limitations on generalization. Multiple comparisons correction was performed the cluster level using AFNI 3dClustSim. Cluster simulation used a non-Gaussian ACF model allowing for heavy tails, using model parameters estimated from the mean of the collected fMRI data using AFNI 3dFWHMx. 


\section{Calculating change in ISC}

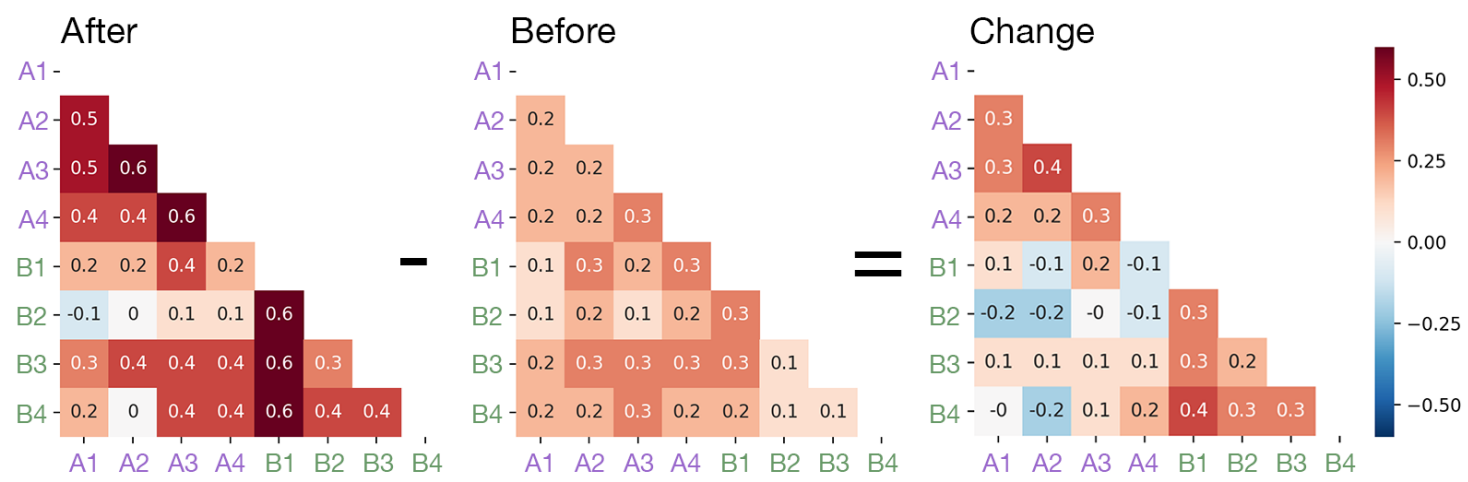

Regression analysis

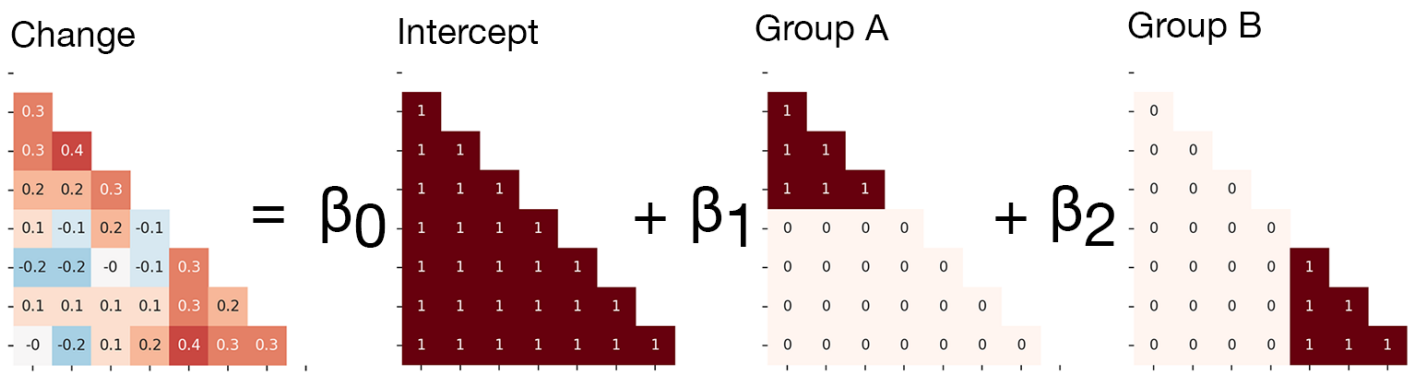

\section{Subject-wise permutation testing}

Original data

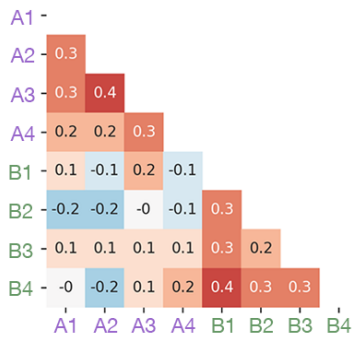

\section{Permuted data}

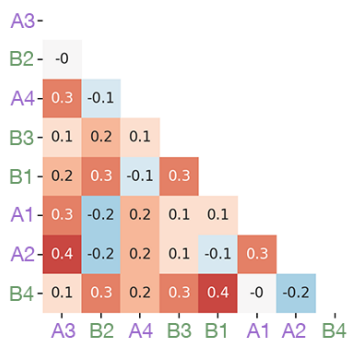

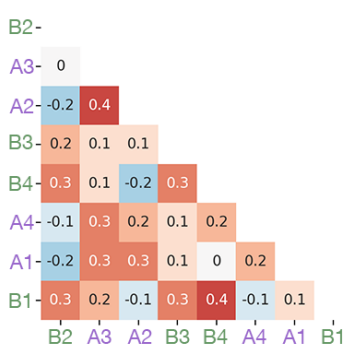

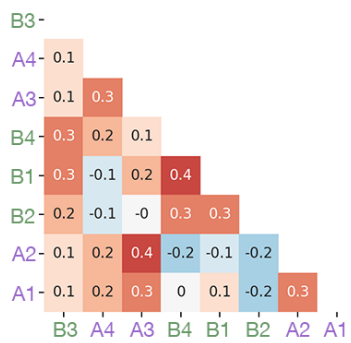

Figure 5: A worked toy example of the change analysis for two groups each containing four participants. Top: ISC matrices were calculated for both fMRI sessions. Each matrix contained ISC measurements for all participant pairs. The before-conversation ISC matrix was subtracted from the after-conversation ISC matrix to obtain the change matrix. Middle: Structure of the multiple regression analysis. Predictor matrices captured change caused simply by watching movie clips twice (the intercept), as well as change caused by conversation within a single group. Bottom: Examples of subject-wise permutation (G. Chen et al., 2016), where rows and columns are identically shuffled.

To identify time points when change occurred, ISC was calculated in a rolling window for each significant cluster in both the before- and after-conversation $\mathrm{fMRI}$ sessions. The rolling window ranged 10 TRs and had a step size of $1 \mathrm{TR}$. The mean pairwise ISC calculated within each window was mapped to the TR corresponding to the window's center, creating a group-level ISC time series. The difference between the before-conversation group ISC time series and the after-conversation group ISC time 
series was used to calculate a group change time series. Group change time series for each significant cluster are included in Appendix 1.

\section{Social network centrality}

Exhaustive maps of participants' social networks were created using an online name generator survey. Participants in this study came from three separate cohorts of MBA students, and separate social networks were generated for each cohort. All first-year students in each cohort (Ns=285, 293, 287; see Figure 7) were emailed a link to the survey website. The sole survey question was adapted from Burt (1992) and read as follows: "Consider the people with whom you like to spend your free time. Since you arrived at [institution name], who are the classmates you have been with most often for informal social activities, such as going out to lunch, dinner, drinks, films, visiting one another's homes, and so on?" To reduce the likelihood of inadequate or biased recall, all possible classmates names were listed alphabetically in four columns, which each column corresponding to one of the MBA program's assigned sections. Participants checked a box next to a name to indicate the presence of a social tie. No limit was placed on the number of social ties or the time taken to complete the survey.

Survey responses were used to create directed graph representations of each network, where nodes corresponded to respondents and edges to social ties. Each cohort had a similar number of social ties/edges $(9472,7340,7676)$ and diameters $(3,3,2)$. Two metrics were computed over these graphs: eigenvector centrality, a measure of how well each node in a graph is connected to other well-connected nodes (Bonacich, 1972), and brokerage, a measure of how well each node connects other nodes that would not otherwise be connected (Burt, 1992) (Figure 6). Both measures were computed using the R package igraph (Csárdi \& Nepusz, 2006). Brokerage was calculated by raising the network's constraint to an exponent of -0.5. Because eigenvector centrality and brokerage are often highly correlated, principal component analysis was used create a centrality measure that captured the variance shared by both metrics. PCA was applied using Scikit-learn (Pedregosa et al., 2012), and the social network metrics were projected on the first principal component. The resulting projection is referred to as $P C A$ centrality. Participants in the fMRI study had widely varying centrality values, covering most of the population distribution (Figure 7). 
A.

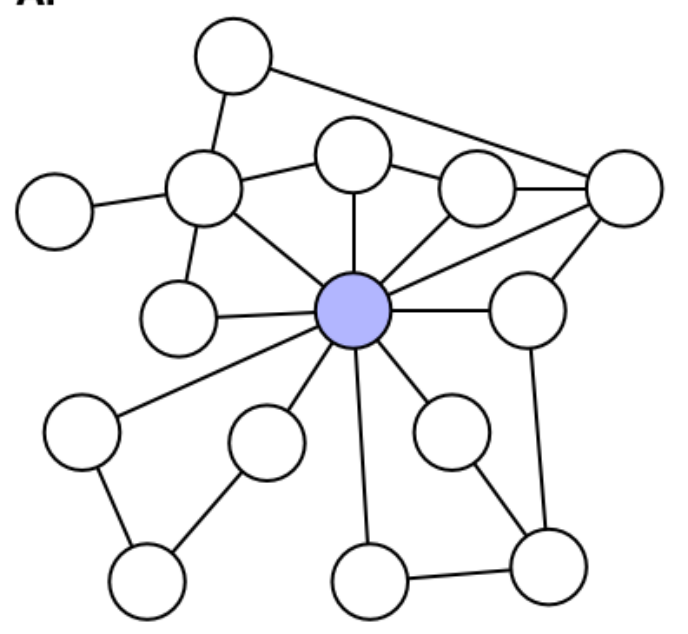

B.

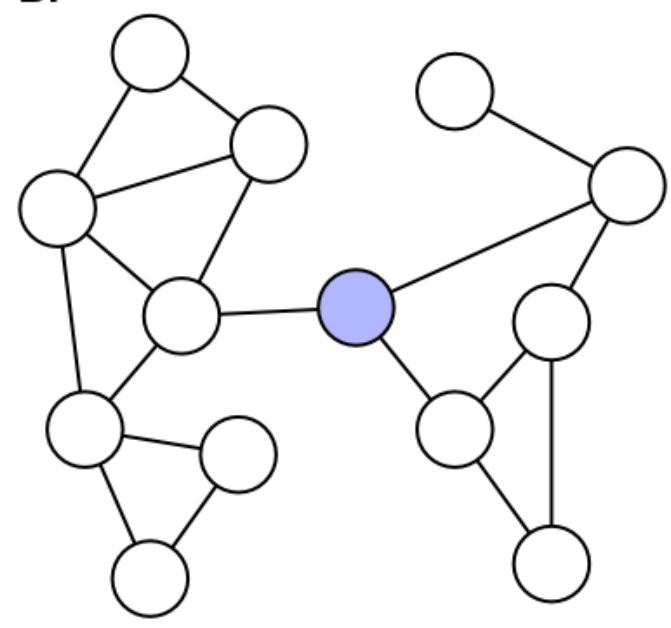

Figure 6: $A$. The highlighted node has high eigenvector centrality-it is connected to many other well-connected nodes. $B$. The highlighted node has high brokerage-it connects many other pairs of nodes that would not otherwise be connected. 


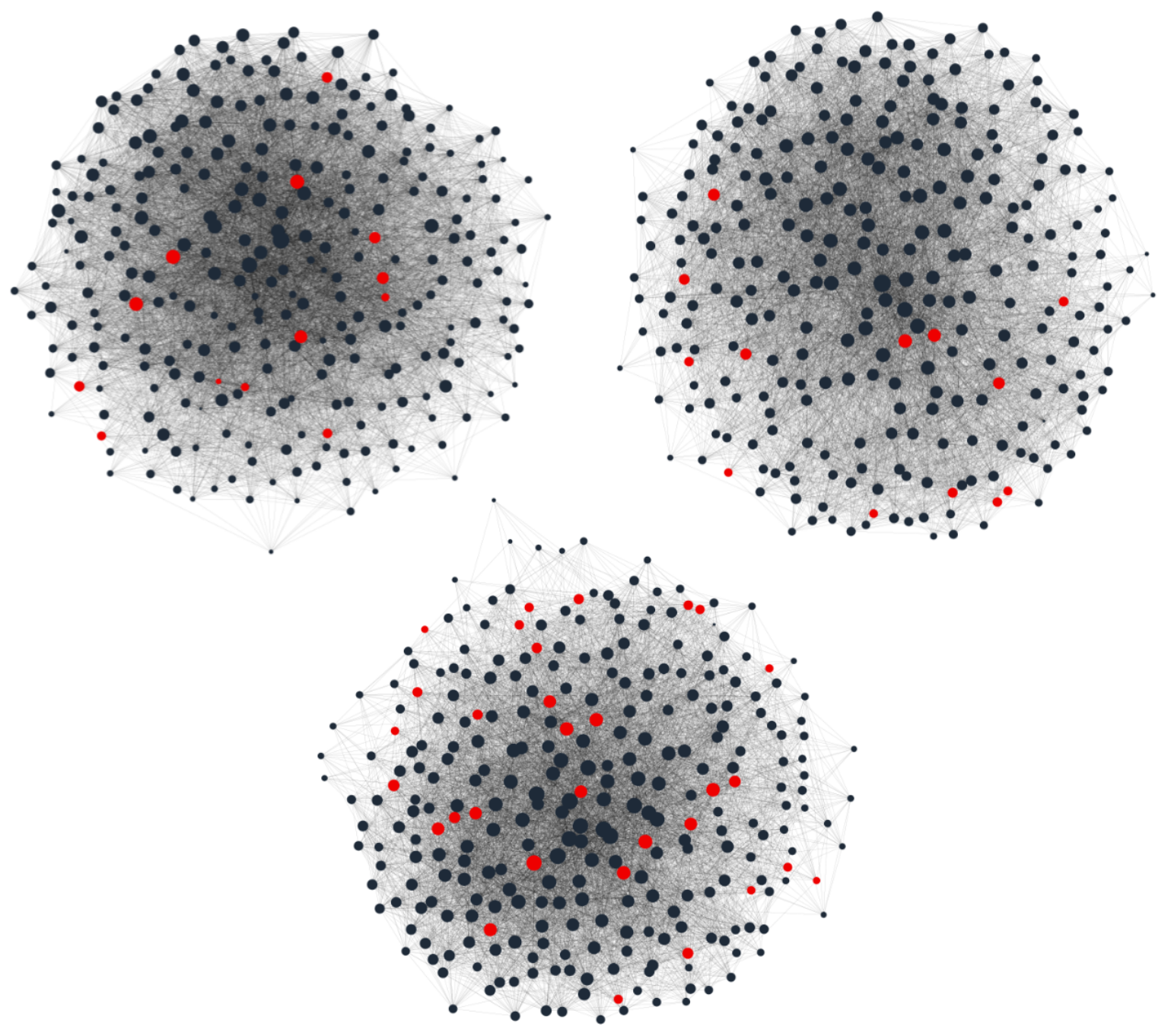

Figure 7: Visualizations of the participants' social networks. Dot size is scaled by PCA centrality (see Methods: Social Network Centrality). Red dots were participants.

\section{Neural influence}

We refer to the participant hypothesized to exert neural influence as the ego, and the participant hypothesized to be influenced as the alter. First, ISC matrices including every possible ego-alter pair were calculated. The initial position matrix measured the ISC of the ego and the alter during the before-conversation $\mathrm{fMRI}$ session, while the final position matrix measured the ISC of the ego before conversation with the alter after conversation. To obtain an influence matrix, the initial position matrix was subtracted from the final position matrix (Figure 8). Unlike the change analysis above, in which only the lower triangle of each matrix was used, in the influence analysis both the upper and lower triangles were used, to test both members of each pair in the ego and the alter position. This procedure yielded neural influence maps at the participant-pair level, with values directly interpretable as how much more similar the alter became to the ego's initial time series.

The effect of social network centrality was assessed using regression analysis over the neural influence maps generated at the level of the participant pair. Predictor matrices were structured similarly to 
the predictors used in the change analysis, but instead of 1s, each cell included the social network metric values of either the ego or the alter. Because predictor beta estimates made with ordinary least squares regression depend only on the variance unique to that predictor, this approach allowed us to separately estimate the effects of PCA centrality, eigenvector centrality, and brokerage on neural influence. This regression yielded maps of centrality-dependent neural influence unique to each movie-group combination.

\section{Calculating neural influence from ISC}

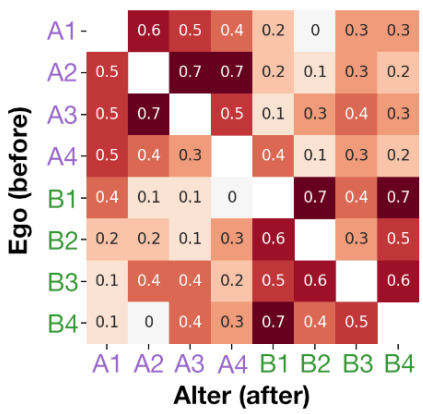

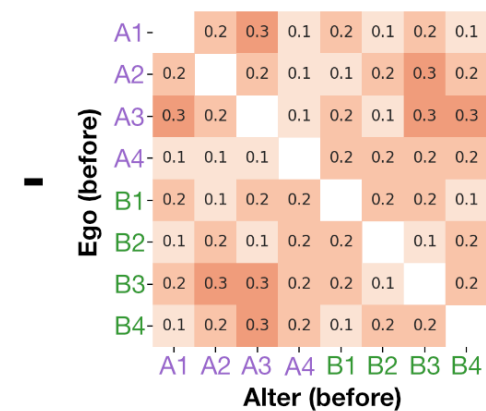

Alter (before)

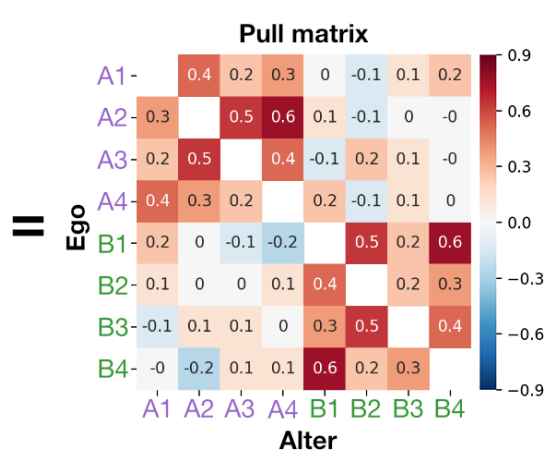

Figure 8: A worked example of how neural influence was calculated based on ISC, for two groups each containing four participants. The influence matrix is determined by assessing how far the alter moved toward the ego's initial position.

The correlation of positive cluster mass in the ego neural influence map with alter ratings of influence during the group conversation for each participant pair was used to test whether neural influence corresponded with social influence.

\section{Reverse inference with Neurosynth}

For each significant region in all of the foregoing analyses and each of the 3,228 Neurosynth terms, we obtained a vector of probabilities that a term would be used, given the spatial pattern of the result (i.e., the posterior probability(feature|activation), assuming an empirical prior). This yielded a matrix where rows corresponded to brain regions and columns to term posterior probabilities. Anatomical and duplicate terms were removed. The columns of the term probability matrix were reduced to 18 components using Principal Component Analysis, capturing $70 \%$ of the variance in the data.

\section{Conversation content analysis}

All conversations were transcribed. Pairs of hypothesis-blind coders classified each speech turn by its function in the conversation. Functional types included: proposing an explanation of the movie clip, accepting an explanation, rejecting (explicit) and rejecting (implicit), editing or changing another's proposal, directing attention to movie content, directing attention to another speaker, giving an order, conversational repair (e.g., "huh?"), and requesting clarification. Coders also rated speaker confidence and deference, whether the speech turn was ignored, whether content in the turn was accepted by the group, whether the turn concerned facts versus opinions, whether the turn referred to other speakers, whether the turn was promped by another speaker, whether the speaker interrupted 
someone, whether others laughed, and whether a joke was intended. Coders also rated participants' perceived social status. Speech turn coding had moderate inter-coder reliability (median Cohen's kappa $=0.53$, all $p$-values $<.001$ ). Ten additional coders rated each participant's perceived social status. Status coding had excellent inter-coder reliability (ICC2 $\mathrm{k}=0.9,95 \% \mathrm{Cl}=[0.86,0.94], \mathrm{F}(38,570)=10.72$, $\mathrm{p}<.001)$. Cohen's kappa for speech turn coding was computed using the i $r \mathrm{r}$ package for $\mathrm{R}$ version 3.6.0 (2019-04-26), and intra-class correlation for status coding was computed using the psych package for $\mathrm{R}$, based on a mean-rating, absolute-agreement, 2-way mixed effects model. Similarly, participants' perceived status values were estimated using beta values from a mixed-effects model with z-scored status ratings as the target, participant ID as the predictor, and random intercepts for rater IDs.

Additional summary measures included included the total number of words spoken by the group (Mahowald, Fedorenko, Piantadosi, \& Gibson, 2013; Wilkes-Gibbs \& Clark, 1992; cf. Cohen Priva, 2017), as well as the the distribution across group members of status, centrality, and words spoken, each using the Gini coefficient (a measure of inequality; Gini, 1955). These predictors were selected to capture leader-follower dynamics (Bales, Strodtbeck, Mills, \& Roseborough, 1951; Dávid-Barrett \& Dunbar, 2012; Gavrilets, Auerbach, \& Van Vugt, 2016; Rueden, Gurven, Kaplan, \& Stieglitz, 2014) and because previous research indicates that high-centrality participants may have particular conversational skills (Celli \& Polonio, 2013; Liu \& Ipe, 2010; Staiano et al., 2012). Because the central tendencies of status and centrality were confounded with their Gini coefficients (status $R=-0.84, p<.001$ ); centrality $R=-$ $0.68, p<.001$ ), central tendency predictors were omitted from the analysis. Accordingly, we describe the the Gini coefficient predictors as simply reflecting the presence of high-status or high-centrality participants in the group.

To account for the hierarchical structure of the data, analyses at the speech turn level included random intercepts for speaker, group, movie, and coder identities. Because our focus was on individual parameters, as opposed to total variance explained, a separate model was trained for each predictor, ensuring variance shared between predictors was represented in the results. P-values were corrected for multiple comparisons using Holm's (1979) sequential procedure. Analysis at the conversation level included a small number of predictors, so a single hierarchical model with all predictors and interactions was trained, with random intercepts for group identity. Models were fitted using the Ime4 package for $\mathrm{R}$ (Bates, Mächler, Bolker, \& Walker, 2015). See Supplementary Table 2 for regression statistics including estimated degrees of freedom.

To analyze conversation content at the word level, all conversation transcripts were preprocessed using Gensim (Rehurek \& Sojka, 2010). This included stripping punctuation, white-space, and numeric values, removing short words and a standard list of stop-words, and word stemming using Porter's (1980) algorithm. Word stems used less than 5 times across all conversations were replaced with the placeholder "RAREWORD." To partially account for the hierarchical structure of the data, word stems were only included in further analysis if they were used by 10 or more participants and in discussions of 3 or more movie clips, excluding participant- and movie- specific terms. 352 word stems were used in the final analysis. A matrix was constructed where rows corresponded to speech turns and columns corresponded to word stem usage counts. Columns were added for the speaker's z-scored PCA centrality, and z-scored perceived status. Using the statsmodels package for Python (Seabold \& Perktold, 2010), separate ordinary least squares regressions were performed with PCA centrality and status as target variables and word stem counts as predictors. Beta values are interpretable as predicted 
increases in the number of standard deviations from the network-wide mean PCA centrality and experiment-wide status values. See Supplementary Table 3 for word-by-word betas and $p$-values.

\section{Data and materials availability}

Unprocessed fMRI data are available in the NIMH Data Archive at http://dx.doi.org/10.15154/1504150.

Stimuli, conversation transcripts, and other materials are available at https://osf.io/kr9fb/.

\section{Code availability}

Code is available on reasonable request.

\section{Acknowledgements}

We thank Robert Burnham developing the scheduling tool, Paulina Calcaterra for assistance collecting data, Margaret Lawson for developing software used during piloting, and Jamil Zaki, Karen Huang, Luke Chang, Jeremy Manning, Janice Chen, Chris Honey, Josh Greene, Peter Tse, and Jim Haxby for insightful discussion. Funding. Project funded by the NIMH of the National Institutes of Health under award number R01MH112566-01 to T. Wheatley and U. Hasson, and Dartmouth seed funding to T. Wheatley and A. Kleinbaum. Author contributions. B. Sievers: Conceptualization, data curation, formal analysis, investigation, project administration, software, visualization, writing - original draft, review \& editing. C. Welker: Investigation, writing - review \& editing. U. Hasson: Conceptualization, funding acquisition, supervision, writing - review \& editing. A. Kleinbaum: Conceptualization, funding acquisition, investigation, resources, supervision, writing - review \& editing. T. Wheatley: Conceptualization, funding acquisition, resources, supervision, writing - review \& editing. Roles defined by the Contributor Roles Taxonomy, available at https://casrai.org/credit/ Competing interests. The authors declare no competing interests.

\section{References}

Andrews, J. A., Tildesley, E., Hops, H., \& Li, F. 2002. The influence of peers on young adult substance use. Health Psychology : Official Journal of the Division of Health Psychology, American Psychological Association, 21(4): 349-357.

Bales, R. F., Strodtbeck, F. L., Mills, T. M., \& Roseborough, M. E. 1951. Channels of Communication in Small Groups. Americal Sociological Review, 16(4): 461-468.

Bates, D., Mächler, M., Bolker, B. M., \& Walker, S. C. 2015. Fitting linear mixed-effects models using Ime4. Journal of Statistical Software, 67(i01). https://doi.org/10.18637/jss.v067.i01.

Berwick, R. C., \& Chomsky, N. 2015. Why Only Us: Language and Evolution. Cambridge, MA: MIT Press. 
Bevilacqua, D., Davidesco, I., Wan, L., Oostrik, M., Chaloner, K., et al. 2018. Brain-to-Brain Synchrony and Learning Outcomes Vary by Student-Teacher Dynamics: Evidence from a Real-world Classroom Electroencephalography Study, 1-11.

Bialic, G., Blichfeld, K., \& Sinclair, B. 2016. "High Maintenance" Tick. Home Box Office.

Bonacich, P. 1972. Factoring and weighting approaches to status scores and clique identification. The Journal of Mathematical Sociology, 2(1): 113-120.

Brennan, S. E., \& Clark, H. H. 1996. Conceptual pacts and lexical choice in conversation. Journal of Experimental Psychology: Learning Memory and Cognition, 22(6): 1482-1493.

Broockman, D., \& Kalla, J. 2016. Durably reducing transphobia: A field experiment on door-to-door canvassing. Science, 3352(6282): 81-87.

Brush, E. R., Krakauer, D. C., \& Flack, J. C. 2018. Conflicts of interest improve collective computation of adaptive social structures. Science Advances, 4(1): 1-10.

Burt, R. D., \& Peterson, A. V. 1998. Smoking cessation among high school seniors. Preventive Medicine, 27(3): 319-27.

Burt, R. S. 1992. Structural holes: the social structure of competition. Cambridge, MA: Harvard University Press.

Burt, R. S. 2010. Neighbour Networks: Competitive Advantage Local and Personal: 410. Oxford, UK: Oxford University Press.

Celli, F., \& Polonio, L. 2013. Relationships between personality and interactions in Facebook. In X. M. Tu, A. M. White, \& N. Lu (Eds.), Social networking: Recent trends, emerging issues and future outlook: 41-53. Hauppauge, NY: Nova Science Publishers.

Chen, G., Shin, Y. W., Taylor, P. A., Glen, D. R., Reynolds, R. C., et al. 2016. Untangling the relatedness among correlations, Part I: Nonparametric approaches to inter-subject correlation analysis at the group level. Neurolmage, 142: 248-259.

Chen, G., Taylor, P. A., \& Cox, R. W. 2016. Is the Statistic Value All We Should Care about in Neuroimaging? bioRxiv. https://doi.org/10.1101/064212.

Chen, J., Leong, Y. C., Honey, C. J., Yong, C. H., Norman, K. A., et al. 2016. Shared memories reveal shared structure in neural activity across individuals. Nature Neuroscience, (December). https: //doi.org/10.1038/nn.4450.

Chen, P. H., White, H. R., \& Pandina, R. J. 2001. Predictors of smoking cessation from adolescence into young adulthood. Addictive Behaviors, 26(4): 517-529.

Chesebro, J. L., \& Martin, M. M. 2003. The relationship between conversational sensitivity, cognitive flexibility, verbal aggressiveness and indirect interpersonal aggressiveness. Communication Research Reports, 20(2): 143-150.

Christakis, N. A. 2004. Social networks and collateral health effects. BMJ (Clinical Research Ed.), 329(7459): 184-185.

Christakis, N. A., \& Fowler, J. H. 2007. The spread of obesity in a social network. The New England Journal of Medicine, 357(4): 370-379. 
Clark, A. 2013. Whatever next? Predictive brains, situated agents, and the future of cognitive science. The Behavioral and Brain Sciences, 36(3): 181-204.

Clark, H. H. 1996. Using Language. Cambridge, UK: Cambridge University Press.

Clark, H. H., \& Brennan, S. E. 1991. Grounding in communication. In L. B. Resnick, J. M. Levine, \& S. D. Teasley (Eds.), Perspectives on socially shared cognition: 127-149. Washington, DC: American Psychological Association.

Cohen Priva, U. 2017. Not so fast: Fast speech correlates with lower lexical and structural information. Cognition, 160: 27-34.

Csárdi, G., \& Nepusz, T. 2006. The igraph software package for complex network research. InterJournal Complex Systems, 1695: 1-9.

Dávid-Barrett, T., \& Dunbar, R. I. M. 2012. Cooperation, behavioural synchrony and status in social networks. Journal of Theoretical Biology, 308: 88-95.

Dean, L. G., Kendal, R. L., Schapiro, S. J., Thierry, B., \& Laland, K. N. 2012. Identification of the social and cognitive processes underlying human cumulative culture. Science, 335(6072): 1114-1118.

Deutsch, M., \& Gerard, H. B. 1955. A study of normative and informational social influences upon individual judgment. The Journal of Abnormal and Social Psychology, 51(3): 629-636.

Dikker, S., Wan, L., Davidesco, I., Bavel, J. J. V., Ding, M., et al. 2017. Brain-to-Brain Synchrony Tracks Real-World Report Brain-to-Brain Synchrony Tracks Real-World Dynamic Group Interactions in the Classroom. Current Biology, 1-6.

Edmondson, A. C., \& Lei, Z. 2014. Psychological Safety: The History, Renaissance, and Future of an Interpersonal Construct. Annual Review of Organizational Psychology and Organizational Behavior, 1(July): 23-43.

Fang, R., Landis, B., Zhang, Z., Anderson, M. H., Shaw, J. D., et al. 2015. Integrating personality and social networks: A meta-analysis of personality, network position, and work outcomes in organizations. Organization Science, 26(4): 1243-1260.

Festinger, L. 1950. Informal social communication. Psychological Review, 57(5): 271.

Feyerabend, P. 1987. Farewell to reason. London: Verso.

Fishkin, J. S. 2019. Democracy When the People Are Thinking: Deliberation and Democratic Renewal. Proceedings of the American Philosophical Society, 163(2): 1-14.

Flack, J. C., Waal, F. B. M. de, \& Krakauer, D. C. 2005. Social structure, robustness, and policing cost in a cognitively sophisticated species. The American Naturalist, 165(5). https://doi.org/10.1086/4292 77.

Fodor, J. A. 1974. Special Sciences (Or: The Disunity of Science as a Working Hypothesis). Synthese, 28(2): 97-115.

Fonov, V., Evans, A. C., Botteron, K., Almli, C. R., McKinstry, R. C., et al. 2011. Unbiased average ageappropriate atlases for pediatric studies. Neurolmage, 54(1): 313-327. 
Friston, K. 2010. The free-energy principle: a unified brain theory? Nature Reviews. Neuroscience, 11(2): 127-38

Garrod, S., \& Pickering, M. J. 2009. Joint Action, Interactive Alignment, and Dialog. Topics in Cognitive Science, 1(2): 292-304.

Gavrilets, S., Auerbach, J., \& Van Vugt, M. 2016. Convergence to consensus in heterogeneous groups and the emergence of informal leadership. Scientific Reports, 6(January): 1-10.

Gini, C. 1955. Variabilità e mutabilità. In E. Pizetti \& T. Salvemini (Eds.), Memorie di metodologica statistica. Rome: Libreria Eredi Virgilio Veschi.

Guntupalli, J. S., Hanke, M., Halchenko, Y. O., Connolly, A. C., Ramadge, P. J., et al. 2016. A Model of Representational Spaces in Human Cortex. Cerebral Cortex, 26(6): 2919-2934.

Hanke, M., Halchenko, Y. O., Sederberg, P. B., Hanson, S. J., Haxby, J. V., et al. 2009. PyMVPA: A python toolbox for multivariate pattern analysis of fMRI data. Neuroinformatics, 7(1): 37-53.

Hasson, U., \& Frith, C. D. 2016. Mirroring and beyond: Coupled dynamics as a generalized framework for modelling social interactions. Philosophical Transactions of the Royal Society B: Biological Sciences, 371(1693). https://doi.org/10.1098/rstb.2015.0366.

Hasson, U., Malach, R., \& Heeger, D. J. 2010. Reliability of cortical activity during natural stimulation. Trends in Cognitive Sciences, 14(1): 40-48.

Hasson, U., Nastase, S. A., \& Goldstein, A. 2019. Robust-fit to nature: an evolutionary perspective on biological (and artificial) neural networks, 1-32.

Holm, S. 1979. Board of the Foundation of the Scandinavian Journal of Statistics A Simple Sequentially Rejective Multiple Test Procedure A Simple Sequentially Rejective Multiple Test Procedure. Source: Scandinavian Journal of Statistics Scand J Statist, 6(6): 65-70.

Honey, C. J., Thesen, T., Donner, T. H., Silbert, L. J., Carlson, C. E., et al. 2012. Slow Cortical Dynamics and the Accumulation of Information over Long Timescales. Neuron, 76(2): 423-434.

Hornsey, M. J. 2008. Social Identity Theory and Self-categorization Theory: A Historical Review. Social and Personality Psychology Compass, 2(1): 204-222.

Huth, A. G., Heer, W. A. D., Griffiths, T. L., Theunissen, F. E., \& Jack, L. G. 2016. Natural speech reveals the semantic maps that tile human cerebral cortex. Nature, 532(7600): 453-458.

Kirchler, E., \& Davis, J. H. 1986. The Influence of Member Status Differences and Task Type on Group Consensus and Member Position Change. Journal of Personality and Social Psychology, 51(1): 83-91.

Lahnakoski, J. M., Glerean, E., Jääskeläinen, I. P., Hyönä, J., Hari, R., et al. 2014. Synchronous brain activity across individuals underlies shared psychological perspectives. Neurolmage, 100: 31624.

Lakoff, G., \& Johnson, M. 1980. Metaphors we live by. Chicago: University of Chicago Press.

Laland, K. N., Uller, T., Feldman, M. W., Sterelny, K., Muller, G. B., et al. 2015. The extended evolutionary synthesis: its structure, assumptions and predictions. Proc Biol Sci, 282(1813): 20151019. 
Landman, B. A., \& Warfield, S. K. (Eds.). 2012. MICCAI 2012 Workshop on Multi-Atlas Labeling (Volume 2). CreateSpace Independent Publishing Platform. https://www.amazon.com/MICCAI-2012Workshop-Multi-Atlas-Labeling/dp/1479126187.

Lazer, D. M. J., Baum, M. A., Benkler, Y., Berinsky, A. J., Greenhill, K. M., et al. 2018. The science of fake news. Science, 359(6380): 1094-1096.

Liu, Y., \& Ipe, M. 2010. How do they become nodes? revisiting team member network centrality. Journal of Psychology: Interdisciplinary and Applied, 144(3): 243-258.

Lupyan, G., \& Clark, A. 2015. Words and the world: Predictive coding and the language-perceptioncognition interface. Current Directions in Psychology, 1-10.

Maass, A., \& Clark, R. D. 1983. Internalization versus compliance: differential processes underlying minority influence and conformity, 13(September 1982).

Magee, J. C., \& Galinsky, A. D. 2008. Social hierarchy: the self-reinforcing nature of power and status. Academy of Management Annals, 2(1): 351-398.

Mahowald, K., Fedorenko, E., Piantadosi, S. T., \& Gibson, E. 2013. Info/information theory: Speakers choose shorter words in predictive contexts. Cognition, 126(2): 313-318.

Martin, M. M., \& Rubin, R. B. 1995. A New Measure of Cognitive Flexibility. Psychological Reports, 76(2): 623-626.

Mednick, S. C., Christakis, N. A., \& Fowler, J. H. 2010. The spread of sleep loss influences drug use in adolescent social networks. PLOS ONE, 5(3). https://doi.org/10.1371/journal.pone.0009775.

Mehra, A., Kilduff, M., \& Brass, D. J. 2001. The social networks of high and low self-monitors: Implications for workplace performance. Administrative Science Quarterly, 46(1): 121-146.

Mroz, J. E., Allen, J. A., Verhoeven, D. C., \& Shuffler, M. L. 2018. Do We Really Need Another Meeting? The Science of Workplace Meetings. Current Directions in Psychological Science, 27(6): 484-491.

Nguyen, M., Vanderwal, T., \& Hasson, U. 2019. Shared understanding of narratives is correlated with shared neural responses. Neurolmage, 184: 161-170.

Parkinson, C., Kleinbaum, A. M., \& Wheatley, T. 2018. Similar neural responses predict friendship. Nature Communications, 9(1). https://doi.org/10.1038/s41467-017-02722-7.

Pattie, C., \& Johnston, R. 2000. "People who talk together vote together": An exploration of contextual effects in Great Britain. Annals of the Association of American Geographers, 90(1): 41-66.

Pattie, C., \& Johnston, R. 2001. Talk as a political context: Conversation and electoral change in British elections, 1992-1997. Electoral Studies, 20(1): 17-40.

Pedregosa, F., Varoquaux, G., Gramfort, A., Michel, V., Thirion, B., et al. 2012. Scikit-learn: Machine Learning in Python, 12: 2825-2830.

Petty, R. E., \& Cacioppo, J. T. 1986. The elaboration likelihood model of persuasion. New York, NY: Springer. https://doi.org/10.1016/S0065-2601(08)60214-2.

Pollack, S., Samuels, S., Fox, J., Orent, K., \& Gilroy, T. 2007. Michael Clayton. Warner Bros. Pictures. 
Pons, V. 2018. Will a five-minute discussion change your mind? A countrywide experiment on voter choice in France. American Economic Review, 108(6): 1322-1363.

Porter, M. F. 1980. An algorithm for suffix stripping. Program, 14(3): 130-7.

Putnam, H. 1967. Psychological Predicates. In W. H. Capitan \& D. D. Merrill (Eds.), Art, mind, and religion: $37-48$. Pittsburgh: University of Pittsburgh Press.

Putnam, H. 1988. Representation and reality. Cambridge, MA: MIT Press.

Rehurek, R., \& Sojka, P. 2010. Software Framework for Topic Modelling with Large Corpora. Proceedings of the LREC 2010 Workshop on New Challenges for NLP Frameworks, 45-50.

Rosenquist, J. N., Fowler, J. H., \& Christakis, N. A. 2011. Social network determinants of depression. Molecular Psychiatry, 16(3): 273-281.

Rueden, C. von, Gurven, M., Kaplan, H., \& Stieglitz, J. 2014. Leadership in an Egalitarian Society. Human Nature, 25(4): 538-566.

Sasovova, Z., Mehra, A., Borgatti, S. P., \& Schippers, M. C. 2010. Network Churn: The Monitoring Personality on Brokerage Dynamics. Administrative Science Quarterly, 55(2010): 639-670.

Seabold, S., \& Perktold, J. 2010. Statsmodels: Econometric and Statistical Modeling with Python. Proceedings of the 9th Python in Science Conference, (Scipy): 92-96.

Simony, E., Honey, C. J., Chen, J., Lositsky, O., Yeshurun, Y., et al. 2016. Dynamical reconfiguration of the default mode network during narrative comprehension. Nature Communications, 7(May 2015): 1-13.

Smith, K. P., \& Christakis, N. A. 2008. Social Networks and Health. Annual Review of Sociology, 34(1): 405-429.

Staiano, J., Lepri, B., Aharony, N., Pianesi, F., Sebe, N., et al. 2012. Friends don't Lie - Inferring personality traits from social network structure. UbiComp'12 - Proceedings of the 2012 ACM Conference on Ubiquitous Computing, 321-330.

Sterelny, K. 2007. Social intelligence, human intelligence and niche construction. Philosophical Transactions of the Royal Society B: Biological Sciences, 362(1480): 719-730.

Sterelny, K. 2010. Minds: extended or scaffolded? Phenomenology and the Cognitive Sciences, 9(4): 465-481.

Tajfel, H., Billig, M. G., Bundy, R. P., \& Flament, C. 1971. Social categorization and intergroup behaviour. European Journal of Social Psychology, 1(2): 149-178.

Taschereau-Dumouchel, V., Cortese, A., Lau, H., \& Kawato, M. 2020. Conducting Decoded Neurofeedback Studies. Social Cognitive and Affective Neuroscience, 0-31.

Theriault, J. E., Young, L., \& Barrett, L. F. 2020. The sense of should: A biologically-based framework for modeling social pressure. Physics of Life Reviews. https://doi.org/10.1016/j.plrev.2020.01.004.

Tylén, K., Weed, E., Wallentin, M., Roepstorff, A., \& Frith, C. D. 2010. Language as a tool for interacting minds. Mind and Language, 25(1): 3-29. 
Wilkes-Gibbs, D., \& Clark, H. H. 1992. Coordinating beliefs in conversation. Journal of Memory and Language, 31(2): 183-194.

Wilson, D., \& Sperber, D. 2002. Relevance Theory. In G. Ward \& L. Horn (Eds.), Handbook of pragmatics. Oxford, UK: Blackwell.

Wuchty, S., Jones, B. F., \& Uzzi, B. 2007. The increasing dominance of teams in production of knowledge. Science, 316(5827): 1036-1039.

Yarkoni, T., Poldrack, R. A., Nichols, T. E., Van Essen, D. C., \& Wager, T. D. 2011. Large-scale automated synthesis of human functional neuroimaging data. Nature Methods, 8(8): 665-670.

Yeshurun, Y., Swanson, S., Simony, E., Chen, J., Lazaridi, C., et al. 2017. Same Story, Different Story: The Neural Representation of Interpretive Frameworks. Psychological Science, 28(3): 307-319.

Zerubavel, N., Hoffman, M. A., Reich, A., Ochsner, K. N., \& Bearman, P. 2018. Neural precursors of future liking and affective reciprocity. Proceedings of the National Academy of Sciences of the United States of America, 115(17): 4375-4380. 